\title{
Infinite wedge and random partitions
}

\author{
Andrei Okounkov
}

\section{Introduction}

The aim of this paper is to show that random partitions have a very natural and direct connection to various structures which are well known in integrable systems. This connection is arguably even more natural than, for example, in the case of random matrices. In a sense, we show that solitaire (which is related to increasing subsequences in random permutations and thus to the Plancherel measure on partitions [2, 3]) and soliton have much more in common than the general notion of solitude.

The other character in the title, the infinite wedge space, is our main technical tool. Starting from the fundamental work of the Kyoto school, this object plays a prominent role in integrable systems.

\section{1}

More concretely, we consider two types of measures on partitions. The first one is the Schur measure $\mathfrak{M}$ for which $\mathfrak{M}(\lambda)$ is proportional to $s_{\lambda}(x) s_{\lambda}(y)$, where $s_{\lambda}$ is the Schur function and $x$ and $y$ are two independent sets of parameters. This measure has a natural representation-theoretic interpretation, see Section 2.1.2. The Plancherel measure is a very particular specialization of $\mathfrak{M}$.

The other measure is the uniform measure which gives equal weight to all partitions of a given number. Our main findings are the following.

\subsection{Schur measure}

We consider the correlation functions of $\mathfrak{M}$, that is, the probability that the set $\mathfrak{S}(\lambda)=\left\{\lambda_{i}-i+\frac{1}{2}\right\}$ contains a given set $X \subset \mathbb{Z}+\frac{1}{2}$. Here the $\frac{1}{2}$ 's are just 
to make certain formulas more symmetric. We prove, see Theorem 1, that

$$
\mathfrak{M}(\lambda, X \subset \mathfrak{S}(\lambda))=\operatorname{det}\left[K\left(x_{i}, x_{j}\right)\right]_{x_{i}, x_{j} \in K},
$$

where the kernel $K$ has a nice generating function, and thus a contour integral representation, in terms of the parameters of the Schur measure $\mathfrak{M}$, see Theorem 2. Note the similarity to situation in random matrices [25], but also observe that $K$ does not involve any objects even remotely as complicated as polynomials orthogonal with respect to an arbitrary measure.

We also prove that, as functions of the parameters of the measure $\mathfrak{M}$, the correlation functions satisfy an infinite hierarchy of PDE's, namely the Toda lattice hierarchy of Ueno and Takasaki [33], see Theorem 3. Again, this is a well known phenomenon in mathematical physics that various correlation functions tend to be $\tau$-functions of integrable hierarchies.

Both of these results are quite straightforward once one interprets the correlation functions as certain matrix elements in the infinite wedge space.

\subsection{Uniform measure}

We give a new, more simple, and conceptual proof of the formula from [5], which is reproduced in Theorem 4 below, for the following averages, called the $n$-point functions,

$$
F\left(t_{1}, \ldots, t_{n}\right)=\sum_{\lambda} q^{|\lambda|} \prod_{k=1}^{n} \sum_{i=1}^{\infty} t_{k}^{\lambda_{i}-i+\frac{1}{2}} .
$$

These $n$-point functions are sums of determinants involving genus 1 theta functions and their derivatives. The key to these $n$-point functions is a system of $q$-difference equations [5] the representation-theoretic derivation of which is given in Theorem 6 .

We also investigate the local structure of a typical large partition and find that, in contrast to the Plancherel measure case [8], it is quite trivial. Locally, a typical partition is a trajectory of a random walk, with probability to make a vertical (resp. horizontal) step depending on the global position on the limit shape.

\subsection{Connections and applications}




\subsubsection{Asymptotic problems}

Our formula for the correlation functions of the Schur measure generalizes the exact formulas for the correlations functions for the Plancherel measure used in [8, 19 to prove the conjecture of Baik, Deift, and Johansson [3] about increasing subsequences in a random permutation and also used in 8] to analyze the local structure of a Plancherel typical partition in the "bulk" of the limit shape. It also generalizes the more general formula of Borodin and Olshanski [10] for the correlation functions of the so called $z$ measures, see Section 2.1.4. The asymptotics of $z$-measures is important for the harmonic analysis on the infinite symmetric group $S(\infty)$, see [9, 23]. Since our kernel $K$ has a simple integral representation, the formula (1.1) is particularly suitable for asymptotic investigations. We also point out that our proofs are considerably simpler and arguably much more conceptual than the ones given in [8, 10].

\subsubsection{Toeplitz determinants and Fredholm determinants}

The results of the present paper were used in [7] to solve a problem proposed, independently, by A. Its and P. Deift. The problem was to find a general identity of the form

$$
\operatorname{det}\left(\phi_{i-j}\right)_{1 \leq i, j \leq n}=\operatorname{det}\left(1-K_{\phi}\right)_{\ell_{2}(\{n, n+1, \ldots\})},
$$

where the kernel $K_{\phi}$ in the Fredholm determinant admits an integral representation in terms of the generating function $\phi(z)=\sum \phi_{n} z^{n}$ for the entries of the Toeplitz determinant. See also [ [- for alternative proofs of the result of [7]. Again, (1.2) seems to be particularly suitable for asymptotic analysis.

\subsubsection{Ramified coverings, moduli spaces, and ergodic theory}

It is known, see e.g. [12], that the Schur and uniform measures on partitions are related to the enumeration of ramified coverings of the sphere and the torus, respectively.

In the case of the torus, the exact formula (3.2) was used in [15] to compute the asymptotics of the number of ramified coverings with given ramification type as the degree goes to infinity. These numbers, which are certain rather complicated polynomials in Bernoulli numbers, can be identified with volumes of certain moduli spaces and are important in ergodic theory [14]. For the coverings of the sphere, see 28]. 


\subsection{Acknowledgments}

I very much benefited from the discussions with S. Bloch, A. Borodin, P. Deift, A. Eskin, M. Kashiwara, S. Kerov, G. Olshanski, Ya. Pugaj, T. Spencer, A. Vershik and others. Much of this paper has grown out of my attempt to better understand the work Borodin, Olshanski, and Kerov on the zmeasures. I would also like to thank NSF for financial support under grant DMS-9801466.

\section{Correlation functions of Schur measure}

\subsection{The Schur measure}

\subsubsection{Definition}

Consider the set of all partitions $\lambda$ and introduce the following function of $\lambda$

$$
\mathfrak{M}(\lambda)=\frac{1}{Z} s_{\lambda}(x) s_{\lambda}(y)
$$

where $s_{\lambda}$ are the Schur functions [24] in auxiliary variables $x_{1}, x_{2}, \ldots$ and $y_{1}, y_{2}, \ldots$, and $Z$ is the sum in the Cauchy identity for the Schur functions

$$
Z=\sum_{\lambda} s_{\lambda}(x) s_{\lambda}(y)=\prod_{i, j}\left(1-x_{i} y_{j}\right)^{-1}
$$

It is clear that if, for exmaple, $\left\{y_{i}\right\}=\overline{\left\{x_{i}\right\}} \subset \mathbb{C}$ and $Z<\infty$ then $\mathfrak{M}$ is a probability measure on the set of all partitions which we shall call the Schur measure. This measure depends on countably many parameters. Since our treatment will be purely algebraic, we shall not require positivity from the measure $\mathfrak{M}$.

\subsubsection{Harmonic analysis interpretation}

It is well known that the Schur functions $s_{\lambda}$ are characters of irreducible representations of the general linear group $G L$ and also encode characters of the symmetric group $S(n)$ as in (2.3) below. Hence one can think of $\mathfrak{M}$ as of a representation-valued measure such that

$$
\mathfrak{M}(\lambda) \propto \lambda \otimes \lambda
$$


where $\boldsymbol{\lambda}$ is a representation of $G L$ or $S(n)$ corresponding to the partition $\lambda$ and $\otimes$ denotes the outer tensor product.

Recall that the classical spaces of noncommutative harmonic analysis

$$
\mathbb{C}[\operatorname{Mat}(n, m)], \quad\left(\mathbb{C}^{n}\right)^{\otimes m}, \quad \mathbb{C}[S(n)]
$$

all decompose into a multiplicity-free direct sum of representations of the form $\boldsymbol{\lambda} \otimes \boldsymbol{\lambda}$ with respect to the natural actions of $G L(n) \times G L(m), G L(n) \times$ $S(m)$, and $S(n) \times S(n)$, respectively. In either of these cases, $\mathfrak{M}(\lambda)$ can be interpreted as the portion of the space which transforms according to the symmetry type $\lambda$.

\subsubsection{Power-sum parameters}

It is convenient to introduce another parameters (also known as the Miwa variables) for the Schur measure

$$
t_{k}=\frac{1}{k} \sum_{i} x_{i}^{k}, \quad t_{k}^{\prime}=\frac{1}{k} \sum_{i} y_{i}^{k}, \quad k=1,2, \ldots
$$

Since the power-sum symmetric functions are free commutative generators of the algebra of the symmetric functions, the Schur function $s_{\lambda}(x)$ is a homogeneous polynomial in the $t_{k}$ 's of degree $|\lambda|$, where

$$
\operatorname{deg} t_{k}=k
$$

More precisely, one has 24

$$
s_{\lambda}(x)=\sum_{\rho=1^{r_{1}} 2^{r_{2}} 3^{r_{3}} \ldots} \chi_{\rho}^{\lambda} \prod_{k} \frac{t_{k}^{r_{k}}}{r_{k} !},
$$

where $\rho=1^{r_{1}} 2^{r_{2}} 3^{r_{3}} \ldots$ means that the partition $\rho$ has $r_{k}$ parts of length $k$ and $\chi_{\rho}^{\lambda}$ is the character of a permutation with cycles $\rho$ in the representation labeled by $\lambda$. Also, we have

$$
Z=\exp \left(\sum_{k>0} k t_{k} t_{k}^{\prime}\right)
$$




\subsubsection{Plancherel measure and $z$-measures}

In particular, if we set

$$
t=t^{\prime}=(\sqrt{\xi}, 0,0, \ldots)
$$

then $\mathfrak{M}$ specializes to the Poissonized Plancherel measure [3, 8, 19]

$$
\mathfrak{M}(\lambda)=e^{-\xi} \xi^{|\lambda|}\left(\frac{\operatorname{dim} \lambda}{|\lambda| !}\right)^{2}
$$

where $\operatorname{dim} \lambda$ is the dimension of the irreducible representation labeled by $\lambda$ and $\xi$ is the parameter of the Poissonization.

The Plancherel measure corresponds to taking just the dimensions of irreducible pieces in the last space in (2.1). Similarly, taking dimensions in the first space in (2.1) and allowing $z=n$ and $z^{\prime}=m$ be arbitrary complex one obtains the $z$-measure.

More precisely, if one sets

$$
t_{k}=\frac{\xi^{k / 2} z}{k}, \quad t_{k}^{\prime}=\frac{\xi^{k / 2} z^{\prime}}{k}, \quad k=1,2, \ldots
$$

where $z, z^{\prime}$, and $\xi$ are parameters, then one obtains the $z$-measure [23, 9, 10]

$$
\mathfrak{M}(\lambda)=(1-\xi)^{z z^{\prime}} \xi^{|\lambda|} s_{\lambda}(\underbrace{1, \ldots, 1}_{z \text { times }}) s_{\lambda}(\underbrace{1, \ldots, 1}_{z^{\prime} \text { times }}) .
$$

Here $s_{\lambda}(\underbrace{1, \ldots, 1}_{z \text { times }})$ stands for the polynomial in $z$ which for positive integer values of $z$ specializes to $s_{\lambda}(x)$ evaluated at $x_{1}=\cdots=x_{z}=1$ and $x_{i}=0$, $i>z$. This polynomial is well known to be 24

$$
s_{\lambda}(\underbrace{1, \ldots, 1}_{z \text { times }})=\prod_{\square \in \lambda} \frac{z+c(\square)}{h(\square)},
$$

where $c(\square)$ is the content of a square $\square \in \lambda$ and $h(\square)$ is its hook-length. More precisely, (2.4) is the mixture of the $z$-measures on partitions of a fixed number $n=|\lambda|$ by the negative binomial distribution on $n=0,1,2, \ldots$ with parameter $\xi$. The Plancherel measure is a limit case of the $z$-measures. 


\subsection{Formula for correlation functions}

\subsubsection{Definition of the correlation functions}

It is convenient to introduce the following coordinates on the set of partitions. To a partition $\lambda$ we associate a subset

$$
\mathfrak{S}(\lambda)=\left\{\lambda_{i}-i+1 / 2\right\} \subset \mathbb{Z}+\frac{1}{2}
$$

For example, $\mathfrak{S}(\emptyset)=\left\{-\frac{1}{2},-\frac{3}{2},-\frac{5}{2}\right\}$. The geometric meaning of the set $\mathfrak{S}(\lambda)$ can be seen from the following picture where the diagram of $\lambda$ is rotated $135^{\circ}$ and the elements of $\mathfrak{S}(\lambda)$ are marked by

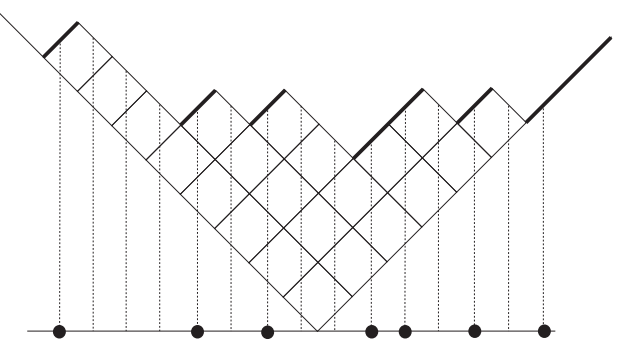

Subsets $S \subset \mathbb{Z}+\frac{1}{2}$ of the form $S=\mathfrak{S}(\lambda)$ can be characterized by

$$
\left|S_{+}\right|=\left|S_{-}\right|<\infty
$$

where

$$
S_{+}=S \backslash\left(\mathbb{Z}_{\leq 0}-\frac{1}{2}\right), \quad S_{-}=\left(\mathbb{Z}_{\leq 0}-\frac{1}{2}\right) \backslash S .
$$

The number $\left|\mathfrak{S}_{+}(\lambda)\right|=\left|\mathfrak{S}_{-}(\lambda)\right|$ is the number of squares in the diagonal of the diagram of $\lambda$ and the finite set $\mathfrak{S}_{+}(\lambda) \cup \mathfrak{S}_{-}(\lambda) \subset \mathbb{Z}+\frac{1}{2}$ is known as the modified Frobenius coordinates of $\lambda$, see [22].

Given a finite subset $X \in \mathbb{Z}+\frac{1}{2}$, define the correlation function

$$
\rho(X)=\mathfrak{M}(\{\lambda, X \subset \mathfrak{S}(\lambda)\}) \text {. }
$$

Our goal in the present section is to prove a determinantal formula for the correlation function

$$
\rho(X)=\operatorname{det}\left[K\left(x_{i}, x_{j}\right)\right]_{x_{i}, x_{j} \in X}
$$

where $K$ is a certain kernel which we shall obtain momentarily. 


\subsubsection{Correlation functions as matrix coefficients}

In what follows, we assume that the reader is familiar with the basics of the infinite wedge space and our notational conventions as summarized for the reader's convenience in the Appendix.

By the definition of the correlation functions $\rho(X)$ and the equations A.16) and (A.1) we have

$$
\rho(X)=\frac{1}{Z} \sum_{\mathfrak{S}(\lambda) \supset X} s_{\lambda}(x) s_{\lambda}(y)=\frac{1}{Z}\left(\Gamma_{+}(t)\left(\prod_{x \in X} \psi_{x} \psi_{x}^{*}\right) \Gamma_{-}\left(t^{\prime}\right) v_{\emptyset}, v_{\emptyset}\right) .
$$

We also have, see (A.10) and (A.11),

$$
\Gamma_{+}(t) v_{\emptyset}=\Gamma_{-}\left(t^{\prime}\right)^{*} v_{\emptyset}=v_{\emptyset}, \quad \Gamma_{+}(t) \Gamma_{-}\left(t^{\prime}\right)=Z \Gamma_{-}\left(t^{\prime}\right) \Gamma_{+}(t) .
$$

From this and (2.5) it follows that

$$
\rho(X)=\left(\prod_{x \in X} \Psi_{x} \Psi_{x}^{*} v_{\emptyset}, v_{\emptyset}\right)
$$

where

$$
\Psi_{k}=G \psi_{k} G^{-1}, \quad \Psi_{k}^{*}=G \psi_{k}^{*} G^{-1}, \quad G=\Gamma_{+}(t) \Gamma_{-}\left(t^{\prime}\right)^{-1}
$$

The presentation (2.6) results in the following

Theorem 1 We have

$$
\rho(X)=\operatorname{det}\left[K\left(x_{i}, x_{j}\right)\right]_{x_{i}, x_{j} \in X},
$$

where $K(x, y)=\left(\Psi_{x} \Psi_{y}^{*} v_{\emptyset}, v_{\emptyset}\right)$.

Proof. The formula (2.7) can be seen as a particular case of Wick's theorem, see e.g. [6]. An equivalent direct combinatorial argument goes as follows. Suppose $|X|=n$ and $X=\left\{x_{1}, \ldots, x_{n}\right\}$. Since $\psi_{x_{i}} \psi_{x_{j}}^{*}=-\psi_{x_{j}}^{*} \psi_{x_{i}}$ for $i \neq j$, the operators $\Psi_{x_{i}}$ and $\Psi_{x_{j}}^{*}$ also anti-commute. Therefore

$$
\rho(X)=\left(\Psi_{x_{1}} \cdots \Psi_{x_{n}} \Psi_{x_{n}}^{*} \cdots \Psi_{x_{1}}^{*} v_{\emptyset}, v_{\emptyset}\right)
$$

Applied to the vacuum $v_{\emptyset}$, the operator $\Psi_{x_{n}}^{*} \cdots \Psi_{x_{1}}^{*}$ removes an $n$-tuple of the vectors $\underline{s}_{i}$ and then the operator $\Psi_{x_{1}} \cdots \Psi_{x_{n}}$ has to put all of them back, in $n$ ! possible orders. Depending on parity of corresponding permutation, these $n$ ! terms appear with a \pm sign and this produces the determinant. 


\subsubsection{Generating function for the kernel $K$}

Form the following generating function

$$
\begin{aligned}
\mathrm{K}(z, w) & =\sum_{i, j \in \mathbb{Z}+\frac{1}{2}} z^{i} w^{-j} K(i, j) \\
& =\left(G \psi(z) \psi^{*}(w) G^{-1} v_{\emptyset}, v_{\emptyset}\right) .
\end{aligned}
$$

Using the formulas (A.12) we compute

$$
G \psi(z) \psi^{*}(w) G^{-1}=\frac{J(z)}{J(w)} \psi(z) \psi^{*}(w),
$$

where

$$
J(z)=\frac{\gamma(z, t)}{\gamma\left(z^{-1}, t^{\prime}\right)}=\exp \left(\sum_{k \geq 1} t_{k} z^{k}-\sum_{k \geq 1} t_{k}^{\prime} z^{-k}\right)=\prod_{i} \frac{1-y_{i} / z}{1-x_{i} z} .
$$

From this and the formula

$$
\left(\psi(z) \psi^{*}(w) v_{\emptyset}, v_{\emptyset}\right)=\sum_{j \in \mathbb{Z}_{\geq 0}+\frac{1}{2}}\left(\frac{w}{z}\right)^{j}=\frac{\sqrt{z w}}{z-w}, \quad|w|<|z|,
$$

we obtain the following

Theorem 2 We have for $|w|<|z|$

$$
\mathrm{K}(z, w)=\frac{\sqrt{z w}}{z-w} \frac{J(z)}{J(w)}
$$

where $J(z)$ is defined by (2.8).

The following results generalize Propositions 2.9 and 2.8 of [8], respectively.

Corollary 1 Define $J_{n}\left(t, t^{\prime}\right)$ by $J(z)=\sum_{n \in \mathbb{Z}} J_{n} z^{n}$. We have

$$
K(i, j)=\sum_{k \in \mathbb{Z}_{\geq 0}+\frac{1}{2}} J_{i+k}\left(t, t^{\prime}\right) J_{-j-k}\left(-t,-t^{\prime}\right) .
$$

Corollary 2 We have $\frac{\partial}{\partial t_{1}} \mathrm{~K}(z, w)=\frac{1}{\sqrt{z w}} \frac{J(z)}{J(w)}$.

In other words, the generating function $\mathrm{K}(z, w)$ factors after taking the partial with respect to $t_{1}$. Similar formulas hold for partials with respect to other parameters. 


\subsection{4 $\quad K$ as an integrable kernel}

Recall that a kernel $K$ is called integrable [18, 11] if the kernel $(x-y) K(x, y)$ has finite rank. In more invariant terms, $K$ is integrable, if its commutator with the operator of multiplication by the independent coordinate has finite rank.

In our case, we have

$$
\begin{aligned}
\sum_{i, j \in \mathbb{Z}+\frac{1}{2}} z^{i} w^{-j}(i-j) K(i, j) & =\left(z \frac{\partial}{\partial z}+w \frac{\partial}{\partial w}\right) \mathrm{K}(z, w)= \\
\frac{\sqrt{z w}}{z-w} & \frac{J(z)}{J(w)}\left(z \frac{\partial}{\partial z}-w \frac{\partial}{\partial w}\right) \log (J(z) J(w)) .
\end{aligned}
$$

Therefore, if the Schur measure is specialized in such a way that

$$
\frac{1}{z-w}\left(z \frac{\partial}{\partial z}-w \frac{\partial}{\partial w}\right) \log (J(z) J(w))=\sum_{i=1}^{N} f_{i}(z) g_{i}(w)
$$

for some $N$ and some functions $f_{1}, \ldots, f_{N}$ and $g_{1}, \ldots, g_{N}$, then $K$ is integrable. This happens if

$$
\frac{\partial}{\partial z} \log J(z) \in \mathbb{C}(z)
$$

where $\mathbb{C}(z)$ stands for the field of rational functions.

\subsection{Remarks}

\subsubsection{Multivariate Bessel functions}

Remark that the functions $J_{n}\left(t, t^{\prime}\right)$ are the most natural multivariate generalization of the classical Bessel functions and specialize to the classical Bessel functions in the Plancherel specialization of the Schur measure [8, 19]. The following picture, where the $z^{4}$ coefficient in $\exp \left[\frac{x}{2}\left(z-z^{-1}\right)+\frac{y}{2}\left(z^{2}-z^{-2}\right)\right]$ is plotted as a function of $x$ and $y$, illustrates how naturally yet nontrivially this bivariate Bessel function interpolates between the classical Bessel functions of order 2 and 4 . 


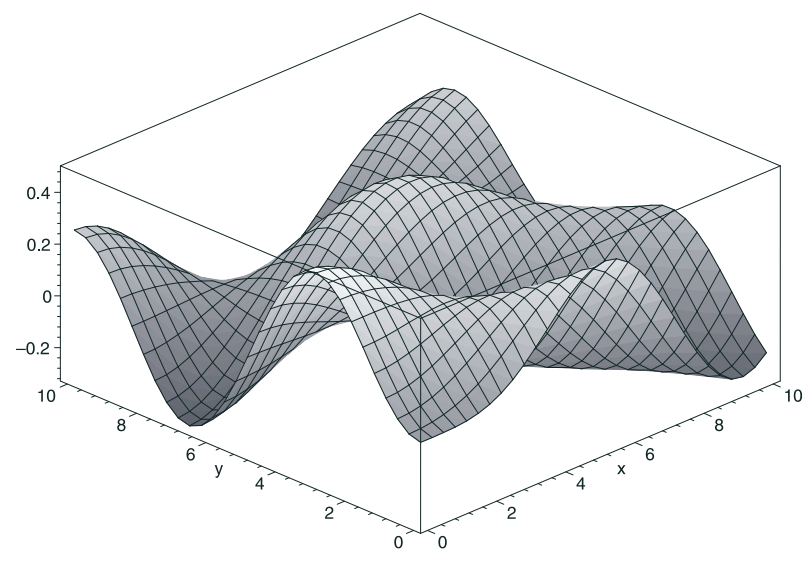

These Bessel functions should not be confused with the (much more complicated) multivariate Bessel functions which arise as suitable limits of the multivariate Jacobi polynomials.

\subsubsection{Toda lattice hierarchy}

The operator $\psi_{k} \psi_{k}^{*}$ satisfies (A.17) and so do the operators

$$
A_{X}=\prod_{x \in X} \psi_{x} \psi_{x}^{*}
$$

Denote by $X+n$ the translation of the set $X$ by an integer number $n$. We have

$$
\begin{aligned}
\left(\Gamma_{+}(t) A_{X} \Gamma_{-}(t) v_{n}, v_{n}\right)=\left(\Gamma_{+}(t)\right. & \left.R^{-n} A_{X} R^{n} \Gamma_{-}(t) v_{\emptyset}, v_{\emptyset}\right) \\
& =\left(\Gamma_{+}(t) A_{X-n} \Gamma_{-}(t) v_{\emptyset}, v_{\emptyset}\right)=Z \rho(X-n)
\end{aligned}
$$

Thus, we have the following

Theorem 3 For any set $X \subset \mathbb{Z}+\frac{1}{2}$ the sequence $\{Z \rho(X-n)\}_{n \in \mathbb{Z}}$ of functions of $t$ and $t^{\prime}$ is a sequence of $\tau$-functions for the Toda lattice hierarchy.

In the particular case when

$$
X=\left\{-\frac{1}{2},-\frac{3}{2},-\frac{5}{2}, \ldots\right\}
$$

these $\tau$-functions are Fredholm determinants with the kernel $K$. The Toda lattice equations for these Fredholm determinants are related to the results of 
[31, 32] and of the recent paper [1]. See [7] for a discussion of the relationship between these Fredholm determinants and Toeplitz determinants.

The times $t$ and $t^{\prime}$ in the Toda lattice hierarchy admit the following combinatorial interpretation. We have

$$
\alpha_{-n} v_{\mu}=\sum_{\lambda / \mu \text { is a rim hook of size } n}(-1)^{\text {height of } \lambda / \mu+1} v_{\lambda}
$$

where by a rim hook we mean a skew diagram $\lambda / \mu$ which is connected and lies on the rim of $\lambda$ and the height of $\lambda / \mu$ is the number of rows it occupies. The action of the operator $\Gamma_{-}\left(t^{\prime}\right)=\prod_{n} \exp \left(t_{n}^{\prime} \alpha_{-n}\right)$ can be interpreted as holding our initial partition for the time $t_{n}$ under a Poisson random stream of $n$-hooks, where each hook attaches to $\alpha$ with a \pm sign according to its height.

Also remark that in the Plancherel case the correlations depend only on the product $t_{1} t_{1}^{\prime}$ and so (A.21) becomes an ODE equivalent to the usual Toda lattice [30].

\subsubsection{Other algebras and Kerov's construction}

Instead of the operators $\alpha_{n}$, which generate an action of the Heisenberg algebra, one can consider representation of other algebras on $\Lambda^{\frac{\infty}{2}} V$. Sandwiching products of $\psi_{k} \psi_{k}^{*}$ between some raising and lowering operators will again produce formulas similar to ours. The analog of the kernel $K$ will involve matrix elements of this representation. In particular, an $\mathfrak{s l}_{2}$-action on $\Lambda^{\frac{\infty}{2}} V$ leads again to $z$-measures and provides another proof of the Borodin-Olshanski formula, see [29]. The action of $\mathfrak{s l}_{2}$ in the basis $\left\{v_{\lambda}\right\}$ gives certain operators on partitions considered by S. Kerov in his analysis of the $z$-measure 21.

\section{The uniform measure}

In this section we shall consider the uniform measure on partitions on $n$ and the related measure

$$
\mathcal{M}_{q}(\lambda)=(q ; q)_{\infty} q^{|\lambda|}, \quad q \in[0,1)
$$

on the set of all partitions. The normalization factor

$$
(q ; q)_{\infty}=(1-q)\left(1-q^{2}\right) \ldots
$$


makes $\mathcal{M}_{q}(\lambda)$ a probability measure; this is clear from the classical identity

$$
\sum_{\lambda} q^{|\lambda|}=\prod_{n \geq 1} \frac{1}{1-q^{n}} .
$$

\subsection{The $n$-point functions}

\subsubsection{Definition}

Again, since our treatment will be purely algebraic, we shall not require $\mathcal{M}_{q}$ to be positive and we shall allow $q$ to be any number from the unit disk $|q|<1$.

Let $f(\lambda)$ be a function on the set of partitions. We set

$$
\langle f\rangle_{q}=(q ; q)_{\infty} \sum_{\lambda} f(\lambda) q^{|\lambda|}
$$

assuming this expectation converges.

Fix an integer $n \geq 1$ and variables $t_{1}, \ldots, t_{n}$. These are not in any way related to the variables $t$ of Section 2. We consider the following $n$-point function

$$
F\left(t_{1}, \ldots, t_{n}\right):=\left\langle\prod_{k=1}^{n}\left(\sum_{i=1}^{\infty} t_{k}^{\lambda_{i}-i+\frac{1}{2}}\right)\right\rangle_{q}
$$

This series converges if $1<\left|t_{i}\right|$ and $\left|t_{1} \cdots t_{n}\right|<|q|^{-1}$ and admits a meromorphic analytic continuation onto $\mathbb{C}^{n}$, see [5] and below. The Laurent coefficients of (3.1) near the point $t_{1}=\cdots=t_{n}=1$ are the averages of the form $\langle f\rangle_{q}$ for polynomial functions $f$ and all averages of polynomials can be reconstructed in this fashion.

\subsubsection{Formula for the $n$-point function}

The following formula for the $n$-point functions was obtained in [5]. Consider the following genus 1 theta function

$$
\begin{aligned}
\Theta(x)=\Theta_{11}(x ; q) & =\sum_{n \in \mathbb{Z}}(-1)^{n} q^{\frac{(n+1 / 2)^{2}}{2}} x^{n+1 / 2} \\
& =q^{1 / 4}\left(x^{1 / 2}-x^{-1 / 2}\right)(q ; q)_{\infty}(q x ; q)_{\infty}(q / x ; q)_{\infty}
\end{aligned}
$$


This is the only odd genus 1 theta function and its precise normalization is not important because the main formula will be homogeneous in $\Theta$. Set

$$
\Theta^{(k)}(x):=\left(x \frac{d}{d x}\right)^{k} \Theta(x ; q) .
$$

We have the following result from [5]

\section{Theorem 4 ([5])}

$$
F\left(t_{1}, \ldots, t_{n}\right)=\sum_{\sigma \in S(n)} \frac{\operatorname{det}\left(\frac{\Theta^{(j-i+1)}\left(t_{\sigma(1)} \cdots t_{\sigma(n-j)}\right)}{\Theta\left(t_{\sigma(1)}\right) \Theta\left(t_{\sigma(1)} t_{\sigma(2)}\right) \cdots \Theta\left(t_{\sigma(1)} \cdots t_{\sigma(n)}\right)}\right)_{i, j=1}^{n}}{(j-i+1) !}
$$

Here $\sigma$ runs through all permutations $S(n)$ of $\{1, \ldots, n\}$, the matrices in the numerator have size $n \times n$, and we define $1 /(-n) !=0$ if $n \geq 1$.

\subsubsection{Strategy of proof}

The proof of (3.2) given in [5] is based on the following two properties of the $n$-point functions. First, these functions satisfy a $q$-difference equation:

Proposition 1 ([5]) We have

$$
\begin{aligned}
& F\left(q t_{1}, t_{2}, \ldots, t_{n}\right)=-q^{1 / 2} t_{1} \ldots t_{n} \times \\
& \sum_{s=0}^{n-1}(-1)^{s} \sum_{1<i_{1}<\cdots<i_{s} \leq n} F\left(t_{1} t_{i_{1}} t_{i_{2}} \cdots t_{i_{s}}, \ldots, \widehat{t_{i_{1}}}, \ldots, \widehat{t_{i_{s}}}, \ldots\right),
\end{aligned}
$$

where hats mean that the corresponding term should be omitted.

Observe that since the $n$-point functions are obviously symmetric, we have parallel formulas for $F\left(t_{1}, \ldots, q t_{k}, \ldots, t_{n}\right)$.

Another property of the $n$-point functions needed in the proof of (3.2) is the description of their singularities. All singularities lie on the divisors

$$
q^{m} t_{i_{1}} t_{i_{2}} \ldots t_{i_{k}}=1, \quad m \in \mathbb{Z}
$$


where $\left\{i_{1}, \ldots, i_{k}\right\} \subset\{1, \ldots, n\}$ is an arbitrary subset. By symmetry, it suffices to consider the divisor $q^{m} t_{1} \ldots t_{k}=1$ on which we have

$$
F\left(t_{1}, \ldots, t_{n}\right)=(-1)^{m} \frac{q^{m^{2} / 2} m^{k-1}}{q^{m} t_{1} \ldots t_{k}-1} \frac{F\left(t_{k+1}, \ldots, t_{n}\right)}{\left(t_{k+1} \ldots t_{n}\right)^{m}}+\ldots
$$

where dots stand for terms regular on the divisor $q^{m} t_{1} \ldots t_{k}=1$ and we assume that

$$
F\left(t_{k+1}, \ldots, t_{n}\right)=1, \quad k=n .
$$

Since we have a $q$-difference equation for the $n$-point functions, it suffices to prove the following

Proposition 2 ([5]) We have

$$
F\left(t_{1}, \ldots, t_{n}\right)=\frac{1}{t_{1}-1} F\left(t_{2}, \ldots, t_{n}\right)+\ldots,
$$

and $F\left(t_{1}, \ldots, t_{n}\right)$ is regular on the divisors $t_{1} t_{2} \cdots t_{k}=1$ for $k>0$.

In this section we shall give a representation-theoretic proof of Propositions 1 and 2 .

\subsection{Singularities of the $n$-point functions}

\subsubsection{Convergence of traces}

Introduce the following operators on $\Lambda^{\frac{\infty}{2}} V$

$$
\begin{gathered}
\mathrm{T}(t)=\sum_{k \in \mathbb{Z}+\frac{1}{2}} t^{k} \psi_{k} \psi_{k}^{*}, \\
: \mathrm{T}(t):=\sum_{k \in \mathbb{Z}+\frac{1}{2}} t^{k}: \psi_{k} \psi_{k}^{*}:
\end{gathered}
$$

where the normal ordering was defined in (A.2). The action of the operator $: \mathrm{T}(t)$ : in the infinite wedge module is well defined for any $t$. The action of $\mathrm{T}(t)$ makes sense only if $|t|>1$ in which case we have

$$
: \mathrm{T}(t):=\mathrm{T}(t)-\sum_{k=-\infty}^{-1 / 2} t^{k}=\mathrm{T}(t)-\frac{1}{t^{1 / 2}-t^{-1 / 2}}
$$


Set $\sigma_{S}=\sum_{k \in S_{+}} t^{k}-\sum_{k \in S_{-}} t^{k}$. It is clear that

$$
: \mathrm{T}(t): f_{S}=\sigma_{S} f_{S}
$$

We have the following estimates

$$
\begin{aligned}
& \left|\sigma_{S}\right| \leq|t|^{\left\|S_{+}\right\|}+\left|S_{-}\right|, \quad|t| \geq 1, \\
& \left|\sigma_{S}\right| \leq|t|^{-\left\|S_{-}\right\|}+\left|S_{+}\right|, \quad|t| \leq 1,
\end{aligned}
$$

where

$$
\|S\|=\sum_{k \in S}|k|
$$

Since

$$
q^{H} f_{S}=q^{\left\|S_{+}\right\|+\left\|S_{-}\right\|} f_{S}
$$

it follows that the following trace

$$
: \mathrm{F}:\left(t_{1}, \ldots, t_{n}\right)=\operatorname{tr}_{\Lambda} \frac{\infty}{2} V\left(q^{H}: \mathrm{T}\left(t_{1}\right):: \mathrm{T}\left(t_{2}\right): \cdots: \mathrm{T}\left(t_{n}\right):\right)
$$

converges absolutely provided

$$
|q|<\left|t_{i_{1}} t_{i_{2}} \ldots t_{i_{k}}\right|<|q|^{-1}
$$

for any subset $\left\{i_{1}, \ldots, i_{k}\right\} \subset\{1, \ldots, n\}$. Let us denote by $\Delta$ this domain of the convergence of (3.6).

\subsubsection{Poles of the $n$-point functions}

Consider the trace

$$
\mathrm{F}\left(t_{1}, \ldots, t_{n}\right)=\operatorname{tr}_{\Lambda} \frac{\infty}{2} V\left(q^{H} \mathrm{~T}\left(t_{1}\right) \mathrm{T}\left(t_{2}\right) \cdots \mathrm{T}\left(t_{n}\right)\right)
$$

which converges absolutely provided $\left|t_{i}\right|>1$ and $\left|t_{1} \cdots t_{n}\right|<|q|^{-1}$. It is clear that using (3.5) one obtains a meromorphic continuation of $F$ onto the domain $\Delta$.

In particular, consider the zero charge subspace $\Lambda_{0} \subset \Lambda^{\frac{\infty}{2}} V$, that is, the kernel of the charge operator $C$. This subspace is preserved by operators $: \mathrm{T}(t)$ : and $H$. It is spanned by the vectors $v_{\lambda}$, where $\lambda$ is a partition, and therefore

$$
F\left(t_{1}, \ldots, t_{n}\right)=(q ; q)_{\infty} \operatorname{tr}_{\Lambda_{0}}\left(q^{H} \mathrm{~T}\left(t_{1}\right) \mathrm{T}\left(t_{2}\right) \cdots \mathrm{T}\left(t_{n}\right)\right) .
$$

In fact, this is the original definition of the $n$-point function in [5]. From the above discussion, we have the following immediate conclusion 
Theorem 5 The $n$-point function $F\left(t_{1}, \ldots, t_{n}\right)$ admits a meromorphic continuation onto the domain $\Delta$ with simple poles along the divisors $t_{i}=1$ and no other singularities. We have

$$
F\left(t_{1}, \ldots, t_{n}\right)=\frac{1}{t_{1}^{1 / 2}-t_{1}^{-1 / 2}} F\left(t_{2}, \ldots, t_{n}\right)+\ldots,
$$

where dots stand for terms regular on the divisor $t_{1}=1$.

\subsection{The $q$-difference equation}

\subsubsection{The effect of the translation operator}

Recall that $R$ denotes the translation operator (A.4). It is clear that

$$
R^{-1} \mathrm{~T}(t) R=t \mathrm{~T}(t) .
$$

Therefore, from (A.5) and (A.6) we obtain

$$
\begin{aligned}
\mathrm{F}\left(t_{1}, \ldots, t_{n}\right) & =\sum_{k} \operatorname{tr}_{R^{k} \Lambda_{0}}\left(q^{H} \mathrm{~T}\left(t_{1}\right) \mathrm{T}\left(t_{2}\right) \cdots \mathrm{T}\left(t_{n}\right)\right) \\
& =\sum_{k} q^{\frac{k^{2}}{2}}\left(t_{1} t_{2} \cdots t_{n}\right)^{k} \operatorname{tr}_{\Lambda_{0}}\left(q^{H} \mathrm{~T}\left(t_{1}\right) \mathrm{T}\left(t_{2}\right) \cdots \mathrm{T}\left(t_{n}\right)\right) \\
& =\Theta_{3}\left(t_{1} t_{2} \cdots t_{n} ; q\right)(q ; q)_{\infty} F\left(t_{1}, \ldots, t_{n}\right)
\end{aligned}
$$

where

$$
\Theta_{3}(t ; q)=\sum_{n \in \mathbb{Z}} q^{\frac{n^{2}}{2}} t^{n}
$$

The function $\Theta_{3}$ satisfies the following $q$-difference equation

$$
\Theta_{3}(q t ; q)=q^{-1 / 2} t^{-1} \Theta_{3}(t ; q) \text {. }
$$

We conclude that the $q$-difference equation (3.3) for the $n$-point function is equivalent to the following equation

$$
\begin{aligned}
& \mathrm{F}\left(q t_{1}, t_{2}, \ldots, t_{n}\right)= \\
& \sum_{s=0}^{n-1}(-1)^{s+1} \sum_{1<i_{1}<\cdots<i_{s} \leq n} \mathrm{~F}\left(t_{1} t_{i_{1}} t_{i_{2}} \cdots t_{i_{s}}, \ldots, \widehat{t_{i_{1}}}, \ldots, \widehat{t_{i_{s}}}, \ldots\right),
\end{aligned}
$$

which will now be established. 


\subsubsection{Proof of the $q$-difference equation}

Note that

$$
\left[\mathrm{T}(t), \psi_{k}^{*}\right]=-t^{k} \psi_{k}^{*}
$$

It follows that

$$
\mathrm{T}\left(t_{1}\right) \cdots \mathrm{T}\left(t_{n}\right) \psi_{k}^{*}=\sum_{P \subset\{1, \ldots, n\}}(-1)^{|P|}\left(\prod_{i \in P} t_{i}^{k}\right) \psi_{k}^{*} \prod_{i \notin P} \mathrm{~T}\left(t_{i}\right) .
$$

Let us assume that $\left|t_{i}\right|>1$ and $\left|t_{1} \cdots t_{n}\right|<q^{-1}$ so that the trace (3.7)

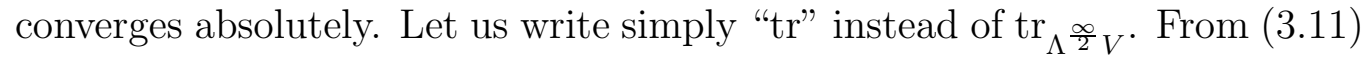
we have

$$
\begin{aligned}
& \operatorname{tr} q^{H} \psi_{k} \mathrm{~T}\left(t_{2}\right) \cdots \mathbf{T}\left(t_{n}\right) \psi_{k}^{*}= \\
& \sum_{P \subset\{2, \ldots, n\}}(-1)^{|P|}\left(\prod_{i \in P} t_{i}^{k}\right) \operatorname{tr} q^{H} \psi_{k} \psi_{k}^{*} \prod_{i \notin P} \mathrm{~T}\left(t_{i}\right) .
\end{aligned}
$$

On the other hand, because of the absolute convergence, we have

$$
\begin{aligned}
\operatorname{tr} q^{H} \psi_{k} \mathrm{~T}\left(t_{2}\right) \cdots \mathrm{T}\left(t_{n}\right) \psi_{k}^{*} & =\operatorname{tr} \psi_{k}^{*} q^{H} \psi_{k} \mathrm{~T}\left(t_{2}\right) \cdots \mathrm{T}\left(t_{n}\right) \\
& =q^{k} \operatorname{tr} q^{H} \psi_{k}^{*} \psi_{k} \mathrm{~T}\left(t_{2}\right) \cdots \mathrm{T}\left(t_{n}\right),
\end{aligned}
$$

where we used the relation $q^{H} \psi_{k}^{*} q^{-H}=q^{-k} \psi_{k}^{*}$. Summing the equations (3.12) we obtain

$$
\begin{aligned}
& \operatorname{tr} q^{H} \widetilde{\mathrm{T}}\left(q t_{1}\right) \mathrm{T}\left(t_{2}\right) \cdots \mathrm{T}\left(t_{n}\right)= \\
& \qquad \sum_{s=0}^{n-1}(-1)^{s} \sum_{1<i_{1}<\cdots<i_{s} \leq n} \mathrm{~F}\left(t_{1} t_{i_{1}} t_{i_{2}} \cdots t_{i_{s}}, \ldots, \widehat{t_{i_{1}}}, \ldots, \widehat{t_{i_{s}}}, \ldots\right),
\end{aligned}
$$

where

$$
\widetilde{\mathrm{T}}(t)=\sum_{k} t^{k} \psi_{k}^{*} \psi_{k}
$$

From the relations

$$
\begin{array}{ll}
\mathrm{T}(t)=: \mathrm{T}(t):+\frac{1}{t^{1 / 2}-t^{-1 / 2}}, & |t|>1, \\
\widetilde{\mathrm{T}}(t)=-: \mathrm{T}(t):-\frac{1}{t^{1 / 2}-t^{-1 / 2}}, & |t|<1,
\end{array}
$$


it follows that

$$
\operatorname{tr} q^{H} \widetilde{\mathrm{T}}\left(q t_{1}\right) \mathrm{T}\left(t_{2}\right) \cdots \mathrm{T}\left(t_{n}\right)=-\mathrm{F}\left(q t_{1}, t_{2}, \ldots, t_{n}\right),
$$

where the right-hand side is computed by analytic continuation. This establishes the following

Theorem 6 The meromorphic function $\mathrm{F}\left(t_{1}, \ldots, t_{n}\right)$ satisfies the following $q$-difference equation

$$
\begin{aligned}
& \mathrm{F}\left(q t_{1}, t_{2}, \ldots, t_{n}\right)= \\
& \sum_{s=0}^{n-1}(-1)^{s+1} \sum_{1<i_{1}<\cdots<i_{s} \leq n} \mathrm{~F}\left(t_{1} t_{i_{1}} t_{i_{2}} \cdots t_{i_{s}}, \ldots, \widehat{t_{i_{1}}}, \ldots, \widehat{t_{i_{s}}}, \ldots\right) .
\end{aligned}
$$

In view of (3.9), this establishes the $q$-difference equation (3.3) and this concludes the proof of Propositions 1 and 2

\subsection{An $\mathcal{M}_{q}$-typical partition is locally a random walk}

The $n$-point functions encode global properties of a measure $\mathcal{M}_{q}$. In this subsection we turn to local properties of an $\mathcal{M}_{q}$-typical partitions and find that their asymptotics is quite trivial. More precisely, as $q \rightarrow 1$, the local structure of an $\mathcal{M}_{q}$-typical partition becomes a random walk with probability to go horizontally or vertically depending on the global position on the rim of the limit shape.

\subsubsection{Correlation functions for Frobenius coordinates}

Recall that the modified Frobenius coordinates of a partition $\lambda$ are defined as follows

$$
\operatorname{Fr} \lambda=\mathfrak{S}(\lambda)_{+} \cup \mathfrak{S}(\lambda)_{-} \subset \mathbb{Z}+\frac{1}{2},
$$

where the set $\mathfrak{S}(\lambda)=\left\{\lambda_{1}-1 / 2, \lambda_{2}-3 / 2, \ldots\right\}$ was defined in (A.7). Given a set $X \subset \mathbb{Z}+\frac{1}{2}$ define the corresponding correlation function by

$$
\begin{aligned}
\varrho(X, q) & =\mathcal{M}_{q}(\{\lambda, X \subset \operatorname{Fr} \lambda\}) \\
& =(q ; q)_{\infty} \sum_{\text {Fr } \lambda \supset X} q^{|\lambda|}
\end{aligned}
$$


here the summation is over all $\lambda$ such that $X \subset \operatorname{Fr} \lambda$.

Recall the following triple product formula for the theta function $\Theta_{3}(z, q)$ defined in (3.10)

$$
\Theta_{3}(z, q)=(q ; q)_{\infty}\left(q^{1 / 2} z ; q\right)_{\infty}\left(q^{1 / 2} / z ; q\right)_{\infty} .
$$

Also recall that one way to prove this triple product formula uses the identity

$$
\left[z^{0}\right]\left(q^{1 / 2} z ; q\right)_{\infty}\left(q^{1 / 2} / z ; q\right)=\sum_{\lambda} q^{|\lambda|}
$$

where the symbol $\left[z^{0}\right]$ in the left-hand side means that we take the constant term in $z$. The combinatorial meaning of (3.13) is the following: on the right, we have all partitions, on the left, we have all possible modified Frobenius coordinates. The same argument establishes the following formula. If $X=$ $\left\{x_{1}, x_{2}, \ldots\right\}$ then

$$
\varrho(X, q)=\left[z^{0}\right]\left(\prod_{x_{i}>0} \frac{q^{x_{i}} z}{1+q^{x_{i}} z} \prod_{x_{i}<0} \frac{q^{-x_{i}} z^{-1}}{1+q^{-x_{i}} z^{-1}} \Theta_{3}(z, q)\right) .
$$

More compactly, this can be written as follows

$$
\varrho(X, q)=\left[z^{0}\right]\left(\prod_{x \in X} \frac{\left(q^{x} z\right)^{\operatorname{sgn}(x) / 2}}{q^{x / 2} z^{1 / 2}+q^{-x / 2} z^{-1 / 2}} \Theta_{3}(z, q)\right) .
$$

Replacing the constant term extraction by an integral we obtain

$$
\varrho\left(X, e^{-2 \pi r}\right)=\int_{-1 / 2}^{1 / 2} \prod_{x \in X} \frac{e^{\operatorname{sgn}(x) \pi(i s-r x)}}{2 \cosh (\pi(i s-r x))} \Theta_{3}\left(e^{2 \pi i s}, e^{-2 \pi r}\right) d s,
$$

where $r>0$.

\subsubsection{Asymptotics of the correlation functions}

Let $N=N(q)$ denote the expected size of a partition with respect to the measure $\mathcal{M}_{q}$

$$
\begin{aligned}
N(q) & =(q ; q)_{\infty} \sum_{\lambda}|\lambda| q^{|\lambda|} \\
& =-q \frac{d}{d q} \log (q ; q)_{\infty}=\frac{1}{24}+G_{2}(q),
\end{aligned}
$$


where $G_{k}(q)=\frac{\zeta(1-k)}{2}+\sum_{n} q^{n} \sum_{d \mid n} d^{k-1}$ is the Eisenstein series. This is, in fact, the simplest example of a polynomial average with respect to $\mathcal{M}_{q}$ which, in the general case, are computed by the formula (3.2). From the quasi-modular property of $G_{2}$ it follows that

$$
N\left(e^{-2 \pi r}\right) \sim \frac{\zeta(2)}{(2 \pi r)^{2}}=\frac{1}{24 r^{2}}, \quad r \rightarrow+0 .
$$

Let us assume that the set $X$ changes with $q \rightarrow 1$ in such a way that all limits

$$
\frac{x_{i}}{\sqrt{N}} \rightarrow \xi_{i}, \quad x_{i} \in X, \quad q \rightarrow 1
$$

exist. That is, we consider the scaling of our typical partition by the factor of $\sqrt{N}$ in both directions, where $N$ is the area of the typical diagram. We are interested in the asymptotics of the correlation functions (3.14). Observe that by our assumption about the growth of $X$ we have

$$
x_{i} r \rightarrow \frac{\xi_{i}}{2 \sqrt{6}}, \quad q=e^{-2 \pi r}, \quad r \rightarrow 0 .
$$

The Jacobi imaginary transformation [17] gives

$$
\Theta_{3}\left(e^{2 \pi i s}, e^{-2 \pi r}\right)=r^{-1 / 2} \sum_{n \in \mathbb{Z}} \exp \left(-\frac{\pi(s-n)^{2}}{r}\right) .
$$

Clearly, as $r \rightarrow+0$, only the $n=0$ summand in the above formula is relevant and the asymptotics of the integral (3.14) is determined by the value of the integrand at $s=0$. Therefore, we obtain the following

Theorem 7 Suppose that, as $q \rightarrow 1$, a finite set $X \subset \mathbb{Z}+\frac{1}{2}$ varies in such a way that all limits

$$
\frac{x_{i}}{\sqrt{N}} \rightarrow \xi_{i}, \quad x_{i} \in X, \quad q \rightarrow 1
$$

exist, where $N=\frac{1}{24}+G_{2}(q)$ is the expectation of $|\lambda|$. Then

$$
\lim _{q \rightarrow 1} \varrho(X, q)=\prod_{x_{i} \in X}\left(1+\exp \left(\frac{\pi\left|\xi_{i}\right|}{\sqrt{6}}\right)\right)^{-1} .
$$


The error term in this formula can be also found by the Laplace method. This theorem says that locally a typical random partition is just a trajectory of a random walk.

Theorem 7 is in agreement with Vershik's theorem [34] about the limit shape of a typical partition with respect to the uniform measure. Namely, Vershik's theorem asserts that after the scaling by the square root of the area in both direction, a typical partition converges to the following limit shape

$$
\exp \left(-\frac{\pi x}{\sqrt{6}}\right)+\exp \left(-\frac{\pi y}{\sqrt{6}}\right)=1
$$

In new coordinates, $u=x-y, v=x+y$ this limit shape becomes

$$
v=\Upsilon(u), \quad \Upsilon(u)=\frac{2 \sqrt{6}}{\pi} \log \left(2 \cosh \left(\frac{\pi u}{2 \sqrt{6}}\right)\right) .
$$

From this we obtain the formula

$$
\frac{1-\left|\Upsilon^{\prime}(u)\right|}{2}=\left(1+\exp \left(\frac{\pi|u|}{\sqrt{6}}\right)\right)^{-1},
$$

which is precisely what we see in the right-hand side of (3.15). See the discussion in in Section 1.3 of [8] for why this should be the case.

\section{A Infinite wedge: summary of formulas}

In this section we collected, for the reader's convenience, some basic formulas related to the infinite wedge space. This material is standard and Chapter 14 of the book [20] can be recommended as a reference. With a few exceptions, we are closely following [20].

\section{Definition}

Let $V$ be a linear space with basis $\{\underline{k}\}, k \in \mathbb{Z}+\frac{1}{2}$. The linear space $\Lambda^{\frac{\infty}{2}} V$ is, by definition, spanned by vectors

$$
v_{S}=\underline{s_{1}} \wedge \underline{s_{2}} \wedge \underline{s_{3}} \wedge \ldots
$$

where $S=\left\{s_{1}>s_{2}>\ldots\right\} \subset \mathbb{Z}+\frac{1}{2}$ is such a subset that both sets

$$
S_{+}=S \backslash\left(\mathbb{Z}_{\leq 0}-\frac{1}{2}\right), \quad S_{-}=\left(\mathbb{Z}_{\leq 0}-\frac{1}{2}\right) \backslash S
$$

are finite. We equip $\Lambda^{\frac{\infty}{2}} V$ with the inner product in which the basis $\left\{v_{S}\right\}$ is orthonormal. 


\section{Free fermions}

Introduce the following operators in $\Lambda^{\frac{\infty}{2}} V$. The operator $\psi_{k}$ is the exterior multiplication by $\underline{k}$

$$
\psi_{k}(f)=\underline{k} \wedge f .
$$

The operator $\psi_{k}^{*}$ is the adjoint operator. These operators satisfy the canonical anti-commutation relations

$$
\psi_{k} \psi_{k}^{*}+\psi_{k}^{*} \psi_{k}=1
$$

all other anticommutators being equal to 0 . It is clear that

$$
\psi_{k} \psi_{k}^{*} v_{S}= \begin{cases}v_{S}, & k \in S, \\ 0, & k \notin S .\end{cases}
$$

Introduce the following generating functions

$$
\psi(z)=\sum_{i \in \mathbb{Z}+\frac{1}{2}} z^{i} \psi_{i}, \quad \psi^{*}(w)=\sum_{j \in \mathbb{Z}+\frac{1}{2}} w^{-j} \psi_{j}^{*} .
$$

and the normally ordered products

$$
: \psi_{k} \psi_{k}^{*}:= \begin{cases}\psi_{k} \psi_{k}^{*}, & k>0 \\ -\psi_{k}^{*} \psi_{k}, & k<0\end{cases}
$$

\section{Energy, charge, and translation operators}

Define the energy and charge operators by

$$
H=\sum_{k} k: \psi_{k} \psi_{k}^{*}:, \quad C=\sum_{k}: \psi_{k} \psi_{k}^{*}:
$$

We have

$$
C v_{S}=\left(\left|S_{+}\right|-\left|S_{-}\right|\right) v_{S}
$$

We will call the operator $R$ defined by

$$
R \underline{s_{1}} \wedge \underline{s_{2}} \wedge \underline{s_{3}} \wedge \cdots=\underline{s_{1}+1} \wedge \underline{s_{2}+1} \wedge \underline{s_{3}+1} \wedge \ldots
$$


the translation operator. In [20], it is denoted by $q$. Clearly

$$
R \psi_{k} R^{-1}=\psi_{k+1}, \quad R \psi_{k}^{*} R^{-1}=\psi_{k+1}^{*} .
$$

It follows that

$$
\begin{aligned}
R^{-k} C R^{k} & =C+k, \\
R^{-k} H R^{k} & =H+k C+\frac{k^{2}}{2} .
\end{aligned}
$$

We have the following charge decomposition

$$
\Lambda^{\frac{\infty}{2}} V=\bigoplus_{k \in \mathbb{Z}} R^{k} \Lambda_{0}
$$

where $\Lambda_{0}=\operatorname{ker} C$ is the zero charge subspace. From (A.3) we see that $\Lambda_{0}$ is spanned by the following vectors

$$
v_{\lambda}=v_{\mathfrak{S}(\lambda)}, \quad \mathfrak{S}(\lambda)=\left\{\lambda_{i}-i+\frac{1}{2}\right\},
$$

where $\lambda$ is a partition. The vacuum vector

$$
v_{\emptyset}=\underline{-\frac{1}{2}} \wedge \underline{-\frac{3}{2}} \wedge-\underline{-\frac{5}{2}} \wedge \ldots
$$

is the vector with the minimal eigenvalue of $H$. The vector

$$
v_{k}=R^{k} v_{\emptyset}=\underline{k-\frac{1}{2}} \wedge \underline{k-\frac{3}{2}} \wedge \underline{k-\frac{5}{2}} \wedge \ldots, \quad k \in \mathbb{Z}
$$

is the vacuum vector in the charge $k$ subspace.

\section{Bosons and vertex operators}

Define the operators $\alpha_{n}$ by

$$
\alpha_{n}=\sum_{k \in \mathbb{Z}+\frac{1}{2}} \psi_{k-n} \psi_{k}^{*}, \quad n= \pm 1, \pm 2, \ldots
$$

They satisfy the Heisenberg commutation relations

$$
\left[\alpha_{n}, \alpha_{m}\right]=n \delta_{n,-m}
$$


see the formula (14.10.1) in [20]. Evidently, $\alpha_{n}^{*}=\alpha_{-n}$. It is clear from definitions that

$$
\left[\alpha_{n}, \psi(z)\right]=z^{n} \psi(z), \quad\left[\alpha_{n}, \psi^{*}(w)\right]=-w^{n} \psi^{*}(w)
$$

Given any sequence $s=\left(s_{1}, s_{2}, \ldots\right)$, define

$$
\Gamma_{ \pm}(s)=\exp \left(\sum_{n=1}^{\infty} s_{n} \alpha_{ \pm n}\right)
$$

Note that this is slightly different from the definition of $\Gamma_{ \pm}$in [20]. We have

$$
\Gamma_{+}(s) v_{m}=v_{m}
$$

Also observe that $\Gamma_{ \pm}^{*}=\Gamma_{\mp}$ and

$$
\Gamma_{+}(s) \Gamma_{-}\left(s^{\prime}\right)=e^{\sum n s_{n} s_{n}^{\prime}} \Gamma_{-}\left(s^{\prime}\right) \Gamma_{+}(s) .
$$

We have from (A.9)

$$
\begin{aligned}
\Gamma_{ \pm}(s) \psi(z) & =\gamma\left(z^{ \pm 1}, s\right) \psi(z) \Gamma_{ \pm}(s) \\
\Gamma_{ \pm}(s) \psi^{*}(z) & =\gamma\left(z^{ \pm 1}, s\right)^{-1} \psi^{*}(z) \Gamma_{ \pm}(s) .
\end{aligned}
$$

Here $\gamma(z, t)$ is the following generating function

$$
\gamma(z, t)=\exp \left(\sum_{n \geq 1} t_{n} z^{n}\right)=\prod_{i} \frac{1}{1-x_{i} z}=\sum_{n \geq 0} z^{n} h_{n}(x)
$$

where the variables $x$ are related to $t$ by (2.2) and the $h_{n}$ 's are the complete homogeneous symmetric functions.

The fermions $\psi(z)$ and $\psi^{*}(z)$ have the following expression in terms of $\Gamma_{ \pm}($see Theorem 14.10 in [20])

$$
\begin{aligned}
\psi(z) & =z^{C} R \Gamma_{-}(\{z\}) \Gamma_{+}\left(-\left\{z^{-1}\right\}\right), \\
\psi^{*}(z) & =R^{-1} z^{-C} \Gamma_{-}(-\{z\}) \Gamma_{+}\left(\left\{z^{-1}\right\}\right),
\end{aligned}
$$

where $\{z\}=\left(z, \frac{z^{2}}{2}, \frac{z^{3}}{3} \ldots\right)$. 


\section{Schur functions as matrix elements}

Using (A.12), one checks that

$$
\left(\Gamma_{-}(t) v_{\mu}, v_{\lambda}\right)=\operatorname{det}\left(h_{\lambda_{i}-\mu_{j}+j-i}(x)\right)=s_{\lambda / \mu}(x),
$$

where $s_{\lambda / \mu}$ is the skew Schur function and the second equality is the JacobiTrudy identity for $s_{\lambda / \mu}$, see [24]. In particular

$$
\Gamma_{-}(t) v_{\emptyset}=\sum_{\lambda} s_{\lambda}(x) v_{\lambda}
$$

\section{Toda lattice and Hirota equations}

Let $A$ be an operator on $\Lambda^{\frac{\infty}{2}} V$ such that

$$
[A \otimes A, \Omega]=0, \quad \Omega=\sum \psi_{k} \otimes \psi_{k}^{*}
$$

Then the following functions of $t$ and $t^{\prime}$

$$
\tau_{n}\left(t, t^{\prime}\right)=\left(\widetilde{A} v_{n}, v_{n}\right), \quad \widetilde{A}=\Gamma_{+}(t) A \Gamma_{-}\left(t^{\prime}\right),
$$

satisfy a hierarchy of Hirota equations. If the operator $A$ satisfies (A.17), then so does $\widetilde{A}$. This commutation equation can be rewritten as

$$
\left[z^{0}\right](\widetilde{A} \otimes \widetilde{A})\left(\psi(z) \otimes \psi^{*}(z)\right)=\left[z^{0}\right]\left(\psi(z) \otimes \psi^{*}(z)\right)(\widetilde{A} \otimes \widetilde{A}),
$$

where $\left[z^{0}\right]$ stands for the constant term in $z$. Taking the matrix coefficients of (A.19) between the vectors

$$
\Gamma_{-}\left(s^{\prime}\right) v_{m} \otimes \Gamma_{-}\left(-s^{\prime}\right) v_{l+1}, \quad \Gamma_{-}(s) v_{m+1} \otimes \Gamma_{-}(-s) v_{l},
$$

and using the equations $(\widehat{A .10}),($ A.11), and (A.14), one obtains the relation

$$
\begin{gathered}
{\left[z^{l-m}\right] \gamma\left(\frac{1}{z},-2 s^{\prime}\right) \tau_{m+1}\left(t+s, t^{\prime}+s^{\prime}+\{z\}\right) \tau_{l}\left(t-s, t^{\prime}-s^{\prime}-\{z\}\right)=} \\
{\left[z^{m-l}\right] \gamma\left(\frac{1}{z}, 2 s\right) \tau_{m}\left(t+s-\{z\}, t+s^{\prime}\right) \tau_{l+1}\left(t-s+\{z\}, t-s^{\prime}\right)}
\end{gathered}
$$

which is satisfied for any $m, l \in \mathbb{Z}$ and any pair of sequences $s$ and $s^{\prime}$. Up to the transformation $t \rightarrow-t$, these are the Hirota equations for the Toda lattice hierarchy of Ueno and Takasaki, see Theorem 1.11 in [33. Expanding 
(A.20) into the Taylor series in $s$ and $s^{\prime}$, one obtains a system of PDE's satisfied by the $\tau_{n}$ 's. In particular, setting $l=m-1$ and extracting the coefficient of $s_{1}^{\prime}$ gives

$$
\frac{\partial^{2}}{\partial t_{1} \partial t_{1}^{\prime}} \log \tau_{n}=\frac{\tau_{n+1} \tau_{n-1}}{\tau_{n}^{2}}
$$

It may be helpful to point out that these Hirota equations are, essentially, Plücker-type relations for the infinite determinants which $\tau$-functions are by their definition. In particular, (A.21) corresponds to the famous determinantal identity which is often associated with Lewis Carroll [13], but was first established by P. Desnanot in 1819, see [26] (I am grateful to A. Zelevinsky for this historical information.)

\section{References}

[1] M. Adler and P. van Moerbeke, Integrals over classical groups, random permutations, Toda and Toeplitz lattices, math.CO/9912143.

[2] D. Aldous and P. Diaconis, Longest increasing subsequences: from patience sorting to the Baik-Deift-Johansson theorem Bull. AMS 36 (1999), no. 4, 413-432.

[3] J. Baik, P. Deift, K. Johansson, On the distribution of the length of the longest increasing subsequence of random permutations, J. Amer. Math. Soc. 12 (1999), no. 4, 1119-1178.

[4] E. Basor and H. Widom, On a Toeplitz determinant identity of Borodin and Okounkov, to appear in Integral Equations and Operator Theory, math.FA/9909010.

[5] S. Bloch and A. Okounkov, The character of the infinite wedge representation, to appear in Adv. Math., alg-geom/9712009.

[6] N. Bogolubov and D. Shirkov, Quantum fields, Benjamin/Cummings Publ., 1983.

[7] A. Borodin and A. Okounkov, A Fredholm determinant formula for Toeplitz determinants, to appear in Integral Equations and Operator Theory, math.CA/9907165. 
[8] A. Borodin, A. Okounkov, and G. Olshanski, On asymptotics of the Plancherel measures for symmetric groups, to appear in J. Amer. Math. Soc., math.CO/9905032.

[9] A. Borodin and G. Olshanski, Point processes and the infinite symmetric group, math.RT/9810015.

[10] A. Borodin and G. Olshanski, Distribution on partitions, point processes, and the hypergeometric kernel, math.RT/9904010.

[11] P. Deift, Integrable operators, AMS Translations, 189, 69-84, 1999.

[12] R. Dijkgraaf, Mirror symmetry and elliptic curves, The Moduli Space of Curves, R. Dijkgraaf, C. Faber, G. van der Geer (editors), Progress in Mathematics, 129, Birkhäuser, 1995.

[13] C. L. Dodgson, The mathematical pamphlets of Charles Lutwidge Dodgson and related pieces, edited by F. F. Abeles, Lewis Carroll Society of North America, Silver Spring, MD, 1994.

[14] A. Eskin and H. Mazur, Pointwise asymptotic formulas on flat surfaces, preprint.

[15] A. Eskin and A. Okounkov, Branched coverings of the torus and volumes of spaces of Abelian differentials, preprint.

[16] I. P. Goulden and D. M. Jackson, Combinatorial enumeration, John Wiley \& Sons, 1983.

[17] Higher Transcendental functions, Bateman Manuscript Project, McGraw-Hill, New York, 1953.

[18] A. R. Its, A. G. Izergin, V. E. Korepin, N. A. Slavnov, Differential equations for quantum correlation functions, Intern. J. Mod. Phys., B4, 1990, 1003-1037.

[19] K. Johansson, Discrete orthogonal polynomials and the Plancherel measure, math.CO/9906120.

[20] V. Kac, Infinite dimensional Lie algebras, Cambridge University Press.

[21] S. Kerov, personal communication. 
[22] S. Kerov and G. Olshanski, Polynomial functions on the set of Young diagrams, C. R. Acad. Sci. Paris Sér. I Math., 319, no. 2, 1994, 121-126.

[23] S. Kerov, G. Olshanski, and A. Vershik, Harmonic analysis on the infinite symmetric group. A deformation of the regular representation, C. R. Acad. Sci. Paris Sér. I Math., 316, no. 8, 1993, 773-778.

[24] I. G. Macdonald, Symmetric functions and Hall polynomials, Clarendon Press, 1995.

[25] M. L. Mehta, Random matrices, Academic Press, 1991.

[26] T. Muir, The theory of determinants, 2nd edition, vol. 1, Macmillan, London, 1906.

[27] A. Okounkov, Random matrices and random permutations, math.CO/9903176.

[28] A. Okounkov, Toda equations for Hurwitz numbers, preprint.

[29] A. Okounkov, $S L(2)$ and the z-measures, preprint.

[30] M. Toda, Theory of nonlinear lattices, Springer-Verlag, 1981.

[31] C. Tracy and H. Widom Random Unitary Matrices, Permutations and Painleve, math.CO/9811154.

[32] C. Tracy and H. Widom, On the Distributions of the Lengths of the Longest Monotone Subsequences in Random Words, math.CO/9904042.

[33] K. Ueno and K. Takasaki, Toda lattice hierarchy, Adv. Studies in Pure Math. 4, Group Representations and Systems of Differential Equations, 1-95, 1984.

[34] A. Vershik, Statistical mechanics of combinatorial partitions and their limit configurations, Func. Anal. Appl., 30, no. 2, 1996, 90-105. 